\title{
Experimentally demonstrate the origin for asymmetric resonance splitting and contributions from couplers to backscattering in SOI microrings
}

\author{
A. Li ${ }^{1}$, T. Van Vaerenbergh ${ }^{1,2}$, P. De Heyn ${ }^{3}$, Y. Xing ${ }^{1}$, P. Bienstman ${ }^{1}$, W. Bogaerts ${ }^{1,4}$ \\ ${ }^{I}$ Photonics Research Group, Department of Information Technology, Center for Nano and Biophotonics, Ghent \\ University imec, Ghent B-9000, Belgium \\ ${ }^{2}$ HP Labs, 1501 Page Mill Road, Palo Alto, CA 94304, USA. \\ ${ }^{3}$ IMEC, Kapeldreef 75, Leuven, Belgium \\ ${ }^{4}$ Also with: Luceda Photonics, 9200 Dendermonde, Belgium \\ ang.li@ugent.be
}

\begin{abstract}
We build a new fitting method which could fit all kinds of resonances of microrings. With that, we experimentally demonstrate the cause for asymmetrically split resonances as well as couplers induced backscattering in SOI microrings.
\end{abstract}

OCIS codes: (230.5750) Resonators; (290.1350) Backscattering; (230.7408) Wavelength filtering devices.

\section{Introduction}

Thanks to its ultra compact size, good tunability and high Q factor, a ring resonator has already become one of the most important building blocks in silicon photonics [1]. It has been intensively used in various applications including sensing, laser cavities, optical filters, optical switches etc. [2-4]. However, backscattering can couple the clockwise $(\mathrm{CW})$ and counter-clockwise circulating $(\mathrm{CCW})$ modes, making the microrings a coupled cavity system rather than a unidirectional cavity. Consequently, rings with sufficiently narrow bandwidth, and a high $\mathrm{Q}$ factor, will have their spectral characteristics distorted due to resonance splitting [5], as shown in figure 1.
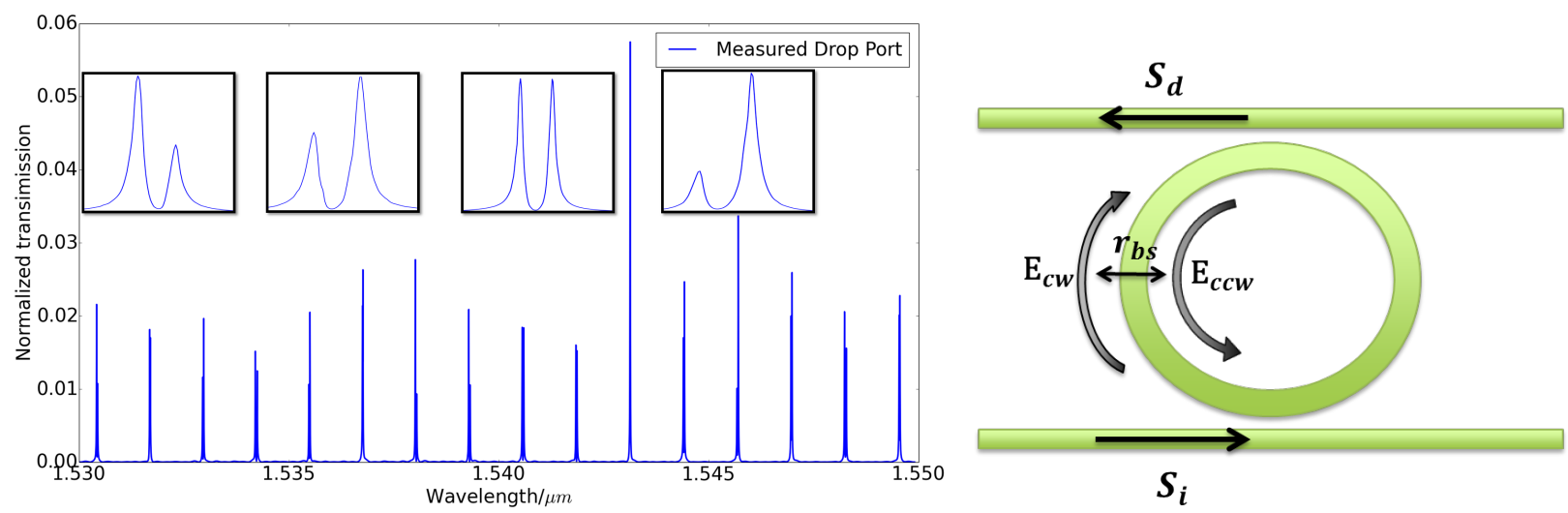

Fig. 1: Left: A measured drop port spectrum of a SOI racetrack ring resonator. Resonances exhibit different splitting, most of which can be asymmetric. The resonance splitting can be attributed to counter-directional coupling in the ring. Right: A schematic of a ring resonator with $\mathrm{CW}$ and $\mathrm{CCW}$ modes coupled by backscattering $r_{b s}$.

There has been significant research in fitting such split resonances and characterizing the contributions to the backscattering. Unfortunately, a model and associated fitting method which is both accurate and robust is still missing and most methods are limited to symmetrically split resonances [6-9], even though most resonances from experiments are asymmetrically split, as also illustrated in figure 1. The research into backscattering focuses largely on the contribution of waveguide roughness, and this cannot explain these asymmetries. 
In this paper, we propose a new ring model and fitting method based on temporal coupled mode theory (tCMT) [10] with backscattering included, which can fit symmetrically and asymmetrically split resonances as well as non-split resonances using the same model. Moreover, for the first time, we experimentally prove the different contributions from the coupling section to backscattering and the reason for asymmetry in split resonances.

\section{Theory and Model}

In our tCMT model, we include both distributed and lumped backscattering. The former one is caused by sidewall roughness along the ring. We assume that the reflected power increases linearly with the ringlength $R_{w}=H_{w} L_{w}$, where $H_{w}$ depends on the waveguide dimension and sidewall quality [11]. The lumped one is attributed to the couplers, it's independent of ringlength, but dependent on coupler parameters. Thus we can write the power reflected by backscattering $R_{b s}=H_{0} L+C_{0}$, where $H_{0} L$ is the contribution from roughness and $C_{0}$ covers the coupler-induced reflection.

On top of the backscattering, we propose the existence of backcoupling $k^{\prime}$ of the directional couplers, i.e. coupling from the in-port to its adjacent port, as $k^{\prime}=f . k$, where $f$ is a dimensionless factor mathematically representing backcoupling as a fraction of coupling coefficient, in order to make backcoupling more tractable and easy to mathematically deal with. Our model will show that this mechanism is responsible for the asymmetry in most split resonances.
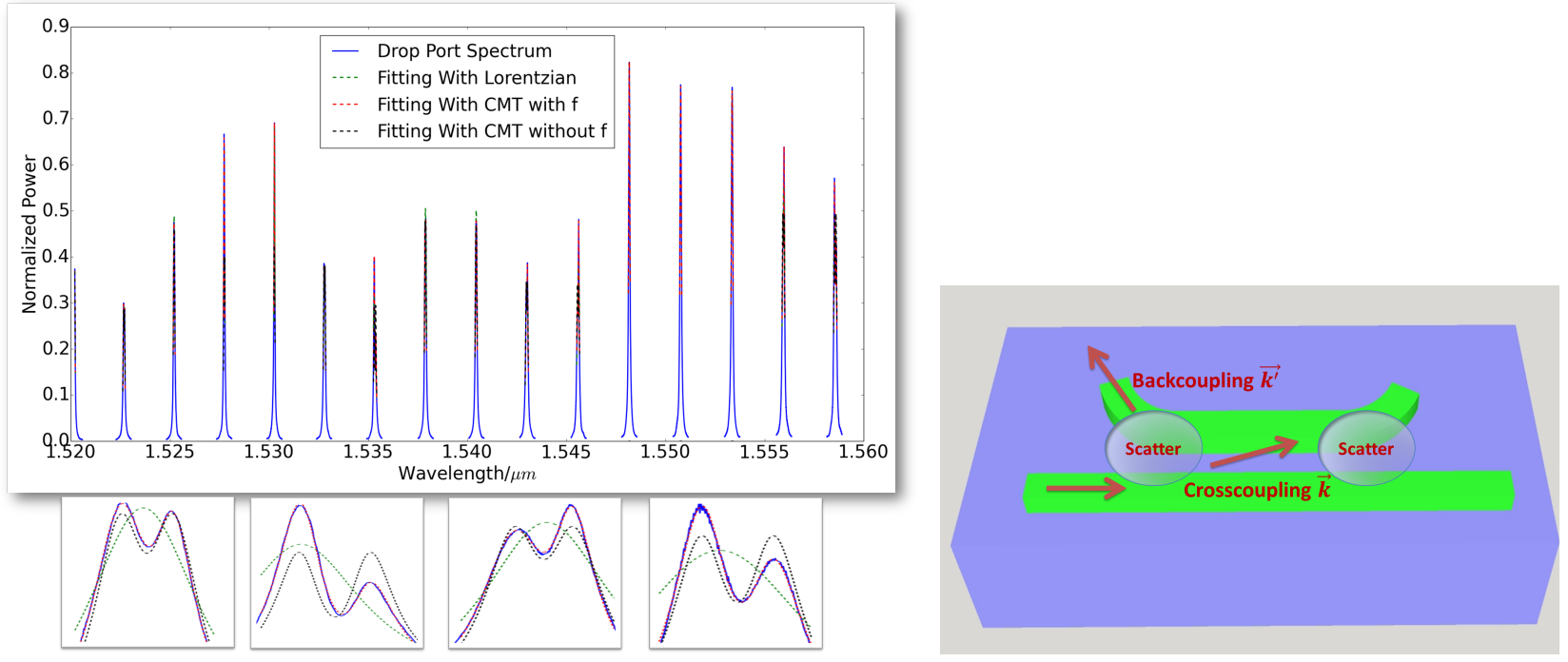

Fig. 2: Left: A comparison of 4 different fitting methods. The blue solid line is the measured data; In green we fit with a traditional Lorentzian; the black line fits with our tCMT model without backcoupling $(f=0)$, and the red line adds the backcoupling, indicated in the right schematic.

We can now derive the amplitude of the wave at the drop port $S_{d}$ for a given input wave $S_{i}, S_{x}$ is normalized such that $S_{x}^{2}$ has units of power :

$$
\left.\frac{S_{d}}{S_{i}}\right|_{b s}=A\left[\frac{(1-f)^{2}}{j\left(\omega-\omega_{1}\right)+\frac{B W_{1}}{2}}+\frac{(1+f)^{2}}{j\left(\omega-\omega_{2}\right)+\frac{B W_{2}}{2}}\right]
$$

A is a amplitude factor, scaled by potential transmission losses. Clearly, there are two resonance modes with their own central frequency $\omega_{1}=\omega_{0}+\mu_{0} \cos \left(\phi_{\mu} / 2\right), \omega_{2}=\omega_{0}-\mu_{0} \cos \left(\phi_{\mu} / 2\right)$ and $3 \mathrm{~dB}$ bandwidth $B W_{1}=B W_{0}+2 \mu_{0} \sin \left(\phi_{\mu} / 2\right)$, $B W_{2}=B W_{0}-2 \mu_{0} \sin \left(\phi_{\mu} / 2\right)$ due to backscattering, where $\omega_{0}, B W_{0}$ are the intrinsic resonance frequency and bandwidth of the ring, and $\mu_{0}, \phi_{0}$ are the amplitude and phase of the mutual coupling of backscattering, respectively. And the $f$ factor, together with the bandwidth, determines the relative power of the two peaks.

\section{Experiments}

Figure 2 compares the fitting using a Lorentzian model with our tCMT model with and without the backcoupling. Our tCMT model fits every resonance quite well, whether it's split or not, symmetric or asymmetric. Also, we find that it is the backcoupling which is indeed responsible for the asymmetry. Even though the bandwidth influences the relative 
power of the two peaks in a split resonances as it stands in the denominator of equation (1) [12], it is frequently shown in our measured spectra that the bandwidths of peaks in split resonances can be extremely similar $\left(\frac{\Delta B W}{B W_{0}}<1 \%\right)$, but power difference could be as large as 50\%, or sometimes peak with larger bandwidth has even higher power, indicating that there must be another factor, i.e. $f$, determining the relative peak power in split resonances.
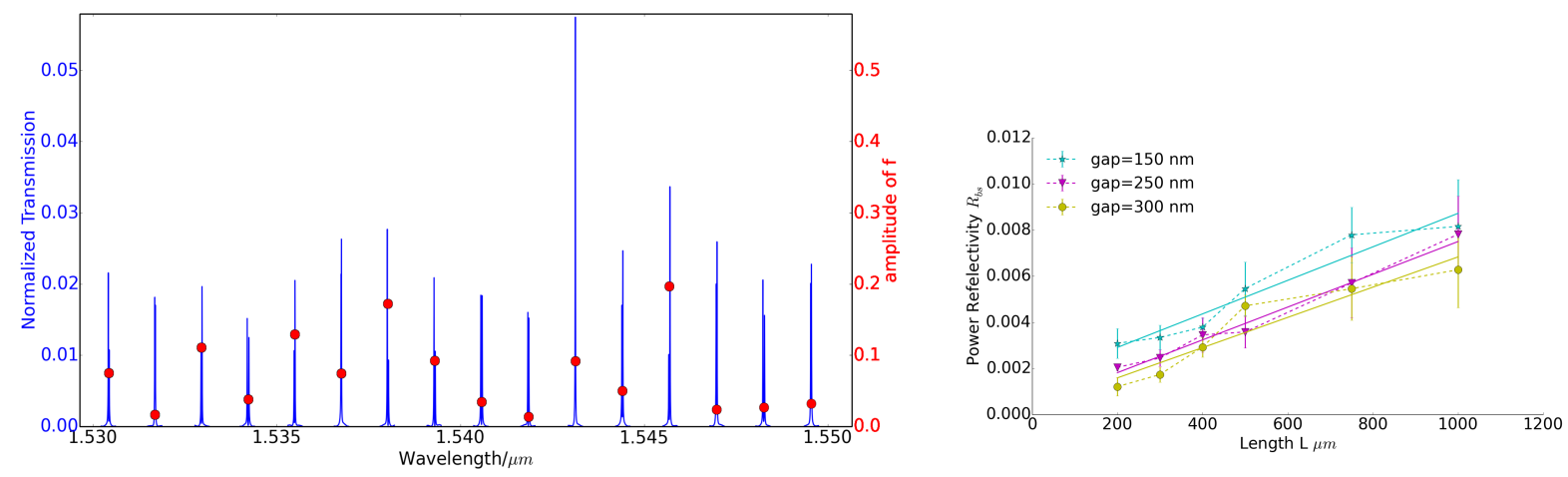

Fig. 3: Left: A measured spectrum with fitted amplitude of $f$ factor at each resonance. Similar in other measured spectra, $f$ is in the range from 0 to 0.4. Right: Fitting to our model over multiple rings. We see an increased backscattering with ringlength $\left(H_{0} L\right)$ and a decreased backscattering with coupler gap $\left(C_{0}\right)$

In Fig. 3, we could find the typical value for the amplitude of $f$ factor. Similar in other measured rings, amplitude of $f$ is in the range of 0-0.4. Consequently, our method claims that the field backcoupled to the adjacent port of the in-port can be as large as $40 \%$ of that coupled to the cross port. right part of Fig. 3 shows the fitting results of our model for $R_{b s}=H_{0} L+C_{0}$, obviously the power reflected by backscattering grows with ring length confirming the waveguide roughness induced backscattering, and for different gap the curve is shifted vertically, indicating the behavior of $C_{0}$. For larger gaps, the backreflection of the directional coupler decrease.

\section{Conclusion}

In summary, we have built a new model and fitting method for ring spectra, capable of explaining all shapes of resonances. Using this model, we have shown that the backcoupling in the couplers is responsible for the asymmetry in split resonances. And the contribution to backscattering from couplers is also proved. These were verified on the measurement data on SOI microrings.

\section{References}

1. W. Bogaerts, P. De Heyn, T. Van Vaerenbergh, K. De Vos, S. Kumar Selvaraja, T. Claes, P. Dumon, P. Bienstman, D. Van Thourhout, and R. Baets, Silicon microring resonators, Laser Photonics Reviews 6, 47-73 (2012).

2. S. T. Chu, B. E. Little, W. Pan, T. Kaneko, S. Sato, and Y. Kokubun, Eight-channel add-drop filter using vertically coupled microring resonators over a cross grid, IEEE Photonics Technology Letters 11, 691-693 (1999).

3. S. Gulde, A. Jebali, and N. Moll, Optimization of ultrafast all-optical resonator switching. Optics express 13, $9502-9515$ (2005).

4. K. De Vos, I. Bartolozzi, E. Schacht, P. Bienstman, and R. Baets, Silicon-on-Insulator microring resonator for sensitive and label-free biosensing. Optics express 15, 7610-7615 (2007).

5. B. E. Little, J. P. Laine, and S. T. Chu, Surface-roughness-induced contradirectional coupling in ring and disk resonators. Optics letters 22 , 4-6 (1997).

6. Z. Zhang, M. Dainese, L. Wosinski, and M. Qiu, Resonance-splitting and enhanced notch depth in SOI ring resonators with mutual mode coupling. Optics express 16, 4621-4630 (2008).

7. M. Moresco, M. Romagnoli, S. Boscolo, M. Midrio, M. Cherchi, E. S. Hosseini, D. Coolbaugh, M. R. Watts, and B. Dutt, Method for characterization of Si waveguide propagation loss. Optics express 21, 5391-400 (2013).

8. G. C. Ballesteros, J. Matres, J. Mart, and C. J. Oton, Characterizing and modeling backscattering in silicon microring resonators, Optics Express 19, 24,980-24,985 (2011).

9. S. Tallur and S. a. Bhave, Rayleigh scattering boosted multi-GHz displacement sensitivity in whispering gallery opto-mechanical resonators, Opt. Express 21, 27,780-27,788 (2013).

10. B. Little, S. Chu, H. Haus, J. Foresi, and J.-P. Laine, Microring resonator channel dropping filters, Journal of Lightwave Technology 15, 998-1005 (1997).

11. F. Morichetti, A. Canciamilla, and A. Melloni, Statistics of backscattering in optical waveguides. Optics letters 35, 1777-1779 (2010).

12. M. A. Popović, Theory and Design of High-Index-Contrast Microphotonic Circuits, Ph.D. thesis (2008). 